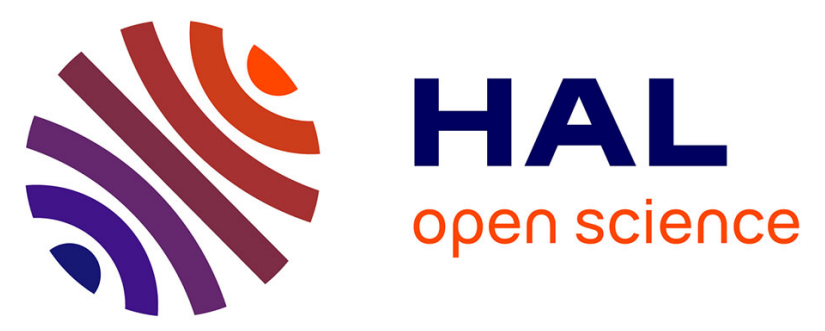

\title{
Correlated optical and electrical analyses of inhomogeneous core/shell InGaN/GaN nanowire light emitting diodes
}

Hezhi Zhang, Valerio Piazza, Vladimir Neplokh, Nan Guan, Fabien Bayle, Stéphane Collin, Ludovic Largeau, Andrey Babichev, Francois H Julien, Maria Tchernycheva

\section{To cite this version:}

Hezhi Zhang, Valerio Piazza, Vladimir Neplokh, Nan Guan, Fabien Bayle, et al.. Correlated optical and electrical analyses of inhomogeneous core/shell InGaN/GaN nanowire light emitting diodes. Nanotechnology, 2020, 10.1088/1361-6528/abc70e . hal-03031214

\author{
HAL Id: hal-03031214 \\ https://hal.science/hal-03031214
}

Submitted on 30 Nov 2020

HAL is a multi-disciplinary open access archive for the deposit and dissemination of scientific research documents, whether they are published or not. The documents may come from teaching and research institutions in France or abroad, or from public or private research centers.
L'archive ouverte pluridisciplinaire HAL, est destinée au dépôt et à la diffusion de documents scientifiques de niveau recherche, publiés ou non, émanant des établissements d'enseignement et de recherche français ou étrangers, des laboratoires publics ou privés. 


\title{
Correlated optical and electrical analyses of inhomogeneous core/shell InGaN/GaN nanowire light emitting diodes
}

\author{
H. Zhang ${ }^{1,2^{*}}$, V Piazza ${ }^{2,3}$, V. Neplokh ${ }^{2,4}$, N. Guan ${ }^{2}$, F. Bayle ${ }^{2}$, S. Collin ${ }^{2}$, L. Largeau ${ }^{2}$, \\ A. Babichev ${ }^{5}$, F. H. Julien ${ }^{2}$ and M. Tchernycheva ${ }^{2}$ \\ ${ }^{1}$ School of Microelectronics, Dalian University of Technology, 116024 Dalian, China \\ ${ }^{2}$ C2N-CNRS, Univ. Paris Saclay, 91120 Palaiseau, France \\ ${ }^{3}$ Ecole Polytechnique Fédérale de Lausanne, 1015 Lausanne, Switzerland \\ ${ }^{4}$ National Research Academic University of the Russian Academy of Sciences, 194021, Saint \\ Petersburg, Russia \\ ${ }^{5}$ ITMO University, 197101, Saint Petersburg, Russia
}

E-mail: hez.zhang@dlut.edu.cn

Received xxxxxx

Accepted for publication $\mathrm{xxxxxx}$

Published xxxxxx

\begin{abstract}
The performance of core-shell InGaN/GaN nanowire light emitting diodes can be limited by wire-to-wire electrical inhomogeneities. Here we investigate an array of core-shell InGaN/GaN nanowires which are morphologically identical, but present electrical dissimilarities in order to understand how the nanoscale phenomena observed in individual nanowires affect the working performance of the whole array. The light emitting diode shows a low number of nanowires ( $20 \%$ ) producing electroluminescence under operating conditions. This is related to a presence of a potential barrier at the interface between the nanowire core and the radially grown n-doped layer, which differently affects the electrical properties of the nanowires although they are morphologically identical. The impact of the potential barrier on the performance of the nanowire array is investigated by correlating multi-scanning techniques, namely electron beam induced current microscopy, electroluminescence mapping and cathodoluminescence analysis. It is found that the main cause of inhomogeneity in the array is related to a non-optimized charge injection into the active region, which can be overcome by changing the contact architecture so that the electrons become injected directly in the n-doped underlayer. The light emitting diode with so-called "front-n-contacting" is developed leading to an increase of the yield of emitting nanowires from $20 \%$ to $65 \%$.
\end{abstract}

Keywords: core-shell nanowire, LED, nitride, EBIC, cathodoluminescence 


\section{Introduction}

Gallium nitride based Nanowires(NWs) appear to be promising to boost the performance of light emitting diodes (LEDs) [1-8], particularly for full-color micro-LEDs [9, 10]. Indeed, three-dimensional nanowire-based LEDs can improve the material quality and offer new opportunities for strain engineering. Moreover, the core-shell architecture is expected to reduce the efficiency droop at high current by increasing the active area. In nanowires (NWs) having their elongated axis aligned with the [0001] direction, quantum confined Stark effect is not present thanks to the growth of the active region on non-polar m-planes [11, 12]. Additionally, the use of nanowires, rather than 2D layers, opens new possibilities for the development of multifunctional light sources. For example, flexible nanowire LEDs were demonstrated by transferring the active nanowires to flexible substrates [13, 14].

The core/shell LEDs were demonstrated using selfassembled growth $[15,16]$ and selective area growth $[17,18]$. Organized growth following the selective area epitaxy procedure provides a better control over the nanowire morphology in comparison with spontaneous growth, since it reduces the composition fluctuations between the NWs and thus facilitates the control of the emission wavelength and the broadening of the spectral emission. However, due to the complexity of the three-dimensional growth it is difficult to control the homogeneity of indium composition inside the quantum well (QW) resulting in compositional gradients with the NW and in the formation of indium-rich regions. In particular, the change of the emission color with the injection current was observed not only in self-assembled NW LEDs [14, 16], but also in organized NW LEDs [19-21]. The transition from a $2 \mathrm{D}$ to a $3 \mathrm{D}$ geometry also affects the electrical and optical properties of the LEDs. Previously, charge carrier trapping [22] has been observed at core/underlayer interface of core-shell NWs creating a potential barrier, which may lead to an inefficient carrier injection towards QWs region. This kind of electrical inhomogeneities can critically affect the LED performance, therefore they deserve a deeper analysis.

In this paper, we investigate core-shell InGaN/GaN NWs by means of macroscopic and nanoscale-resolved techniques. A sample which shows a good morphological order, but exhibits inhomogeneities of the electroluminescent (EL) was deliberately chosen. First, the morphology and the internal structure of the NWs is analyzed, together with the EL of the array. Cross-sectional electron beam induced current microscopy (EBIC) is performed, revealing a potential barrier induced by charge carrier traps at the core/underlayer interface. Then, the impact of this potential barrier on the performance of the InGaN/GaN NW LED is statistically analyzed, by correlating top view EBIC, cathodoluminescent
(CL) and EL maps. Finally, we developed an alternative processing scheme to overcome this issue by injecting the electrons through $\mathrm{n}-\mathrm{GaN}$ underlayer thus avoiding the potential barrier. The final device shows a significant improvement in the EL homogeneity of the NW LEDs.

\section{Nanowire growth, device fabrication and preliminary analysis}

Organized InGaN/GaN core-shell NWs are grown by Metalorganic Vapour-Phase Epitaxy (MOVPE) on n-type $\mathrm{GaN} /$ sapphire substrates coated with a $\mathrm{SiN}_{\mathrm{x}}$ mask with submicrometer openings. First, n-type GaN core is grown along the c-direction, folowing the lateral overgrowth of $n$-doped $\mathrm{GaN}$ underlayer, which is overgrown with a single InGaN QW. The structure is completed with a magnesium doped ptype cap layer consisting of an AlGaN electron blocking layer and a $\mathrm{p}-\mathrm{GaN}$ shell. Figure 1(a) shows a schematic of the internal structure of an individual nanowire in the LED. The NWs consist of a base part, which has the form of a hexagonal cylinder defined by m-planes of about $1 \mu \mathrm{m}$ in height and of a pyramidal top part defined by semi-polar facets. The NW ensemble presents a high morphological wire-to-wire homogeneity as seen from the SEM image of Figure 1(b). However, due to SiN mask imperfections, some of the NWs in the pattern are missing (e.g. an empty place can be seen in the right part of Figure 1(b)). The quantity of missing wires amounts to $\sim 5-8 \%$, no other morphological defects were observed. These pattern defects are used in the present work to facilitate correlation between different maps as discussed in the following.

The NW structure is confirmed by scanning transmission electron microscopy (STEM) as illustrated in Figure 1(c). The growth on the semipolar plane is almost inhibited (cf. Figure 1(d)), so that the thickness of the InGaN QW on the pyramidal facets is below $1 \mathrm{~nm}$ and it does not patriciate in the EL emission [23]. However, an indium-rich region is formed at the junction between the m-plane and the semi-polar plane, as confirmed by the Energy-dispersive X-ray diffraction (EDX) measurements shown in Figure 1(d).

In order to test the nanowire array, the sample was processed into standard $300 \times 300 \mu \mathrm{m}^{2}$ size LED mesas. The Indium tin oxide (ITO)-covered mesas were defined by optical lithography and a metal frame $(\operatorname{Ti}(10 \mathrm{~nm}) / \mathrm{Au}(150 \mathrm{~nm}))$ was deposited on the top surface. To obtain the bottom contact, Inductively Coupled Plasma Etching (ICP) etching was used to remove partially the nanowires in order to access the $n-G a N$ buffer layer from the top where a $\operatorname{Ti}(10 \mathrm{~nm}) / \mathrm{Al}(30 \mathrm{~nm}) / \operatorname{Ti}(10$ $\mathrm{nm}) / \mathrm{Au}(150 \mathrm{~nm})$ metallization was applied.

Under operating condition, the LED produces luminescence starting from a bias of $3.3 \mathrm{~V}$. An example of the EL spectrum and the corresponding optical image of the LED is shown in Figure 1(e)) for electrical power of $1.1 \mathrm{~mW}$. Similarly to our previous report [23], the spectrum presents 
two contributions: the high-energy peak at $2.6 \mathrm{eV}(477 \mathrm{~nm})$ arises from the m-plane QW and the low-energy peak at 2.4 $\mathrm{eV}(517 \mathrm{~nm})$ is attributed to the emission from the indium-rich region at the m-plane/semi-polar plane junction. For further details on the electrical and optical properties, please refer to our previous publications $[19,22,23]$.
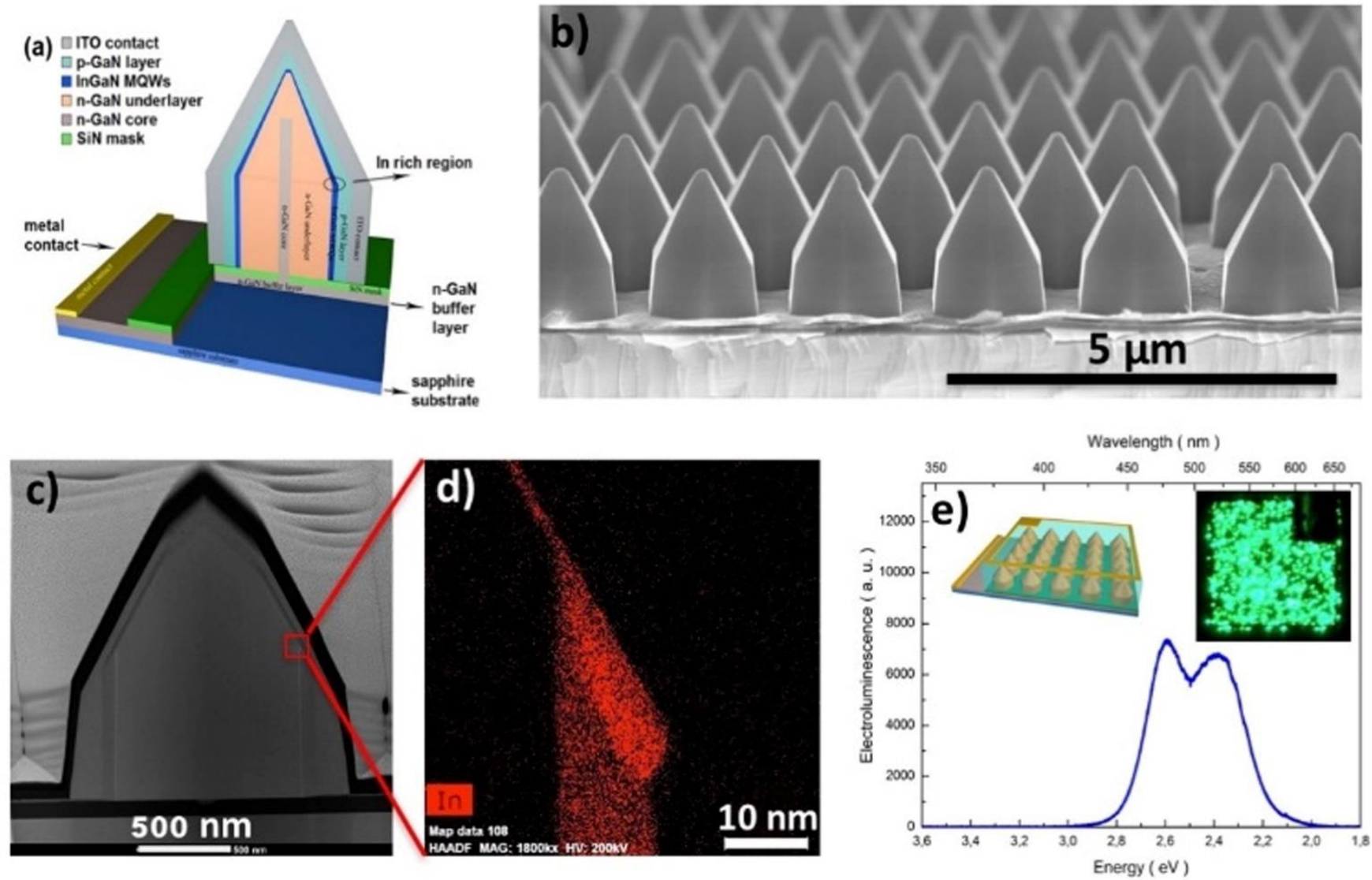

Figure 1. (a) Schematic of a cleaved NW showing its internal structure. (b) $30^{\circ}$ tilted SEM image of the ITO-covered NWs. (c) Cross-sectional STEM image of a NW and (d) the EDX map showing the indium rich region in the QW at the junction between the m-plane and the semi-polar plane. (e) Electroluminescence spectrum of the LED. The insets show a schematic representation of the mesa and an optical microscope photo of the LED under operation.

In addition, the EL map shows that only a small percentage of NWs are emitting light in operation conditions. Based on high magnification images (Figure $8(\mathrm{a})$ ), this percentage is estimated to be in the range of $20 \%-28 \%$, depending on the applied external bias (3.3-5.0 V). The spotty nature of the EL map may have different origins such as the difference in the electrical properties changing the local current injection or the wire-to-wire variation of material properties (e.g. some wiredependent non-radiative defects affecting the internal quantum efficiency). In order to understand which factor impacts the most the homogeneity of the array, we performed a multi-technique analysis on the same area by means of EBIC, EL and CL, as described in the following.

\section{Cross-sectional EBIC characterization of individual cleaved nanowires}

First, the electrical properties of individual NWs are studied by cleaving the vertical structures as shown in the SEM image of Figure 2(a). The EBIC maps are acquired at room temperature in a Hitachi SU8000 SEM controlled by a Gatan DigiScan system as described in [24]. The experiments are performed under $10 \mathrm{kV}-15 \mathrm{kV}$ acceleration voltage and $130-200 \mathrm{pA}$ impinging current.

As expected, the majority of the cleaved NWs presented an EBIC signal at the p-n junction position in agreement with the previous observations on similar structures [23]. However, several cleaved NWs showed a different behaviour: first, a weak signal appeared at the interface between the $n-G a N$ core and the $\mathrm{n}-\mathrm{GaN}$ underlayer. An example of this abnormal EBIC signal is shown in Figure 2(b), where it can be well observed as a narrow stripe in the bottom part of the NW, which gradually broadens toward the upper part. Under electron beam irradiation of the wire for about $10 \mathrm{~min}$, the EBIC map was modified: the core/underlayer signal disappeared and transformed into a conventional signal from the $\mathrm{p}-\mathrm{n}$ junction (Figure 2(c)). The abnormal EBIC signal was observed in 
several wires. In particular, NWs cleaved almost perpendicular to the $\mathrm{c}$ axis also presented this behaviour (cf. Figure 2(d) and (e)). Top view EBIC measurements on NW arrays discussed further have also evidenced the abnormal EBIC signal allowing to obtain a statistical information about the frequency of its occurrence.

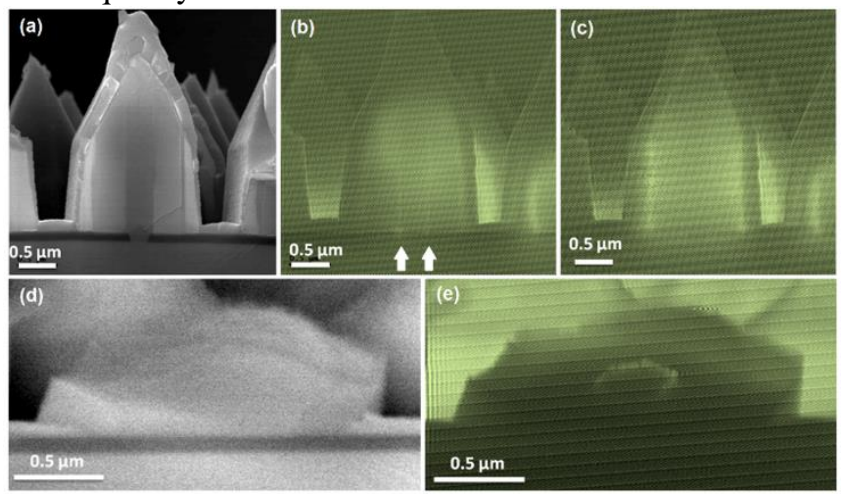

Figure 2. (a) SEM image at $\mathrm{V}_{\mathrm{acc}}=10 \mathrm{kV}$ of a nanowire cleaved along its axis. (b) Corresponding EBIC map before transformation. The white arrows show the position of the abnormal signal. (c) EBIC map of the same region after transformation. (d) SEM image at $\mathrm{V}_{\mathrm{acc}}=15 \mathrm{kV}$ of a nanowire cleaved almost perpendicularly to its axis. (e) Corresponding EBIC map showing the abnormal signal at the core / underlayer interface.

The transformation of the abnormal signal into the p-n junction signal was permanent: the EBIC activity at the core/underlayer interface could be observed only during first minutes of exposure. When observing the same NW after several hours in the dark, only the p-n junction signal was present.

This kind of behaviour has been previously reported in core/shell NW LEDs [22]. It was tentatively ascribed to a parasitic potential barrier at the core/underlayer interface. One possible origin of this potential barrier may be related to a strong silicon doping of the $\mathrm{n}-\mathrm{GaN}$ core.

Indeed, in 2D GaN layers, silicon was identified as an antisurfactact which may induce partial masking of the GaN growth surface by $\mathrm{Si}-\mathrm{N}$ bonds [25] or by $\mathrm{Si}_{3} \mathrm{~N}_{4}$ islands [26] or even by a $\mathrm{SiGaN}_{3}$ monolayer [27]. In the NW case, for heavily Si-doped GaN NWs grown by MOVPE (doping exceeding $10^{20} \mathrm{~cm}^{-3}$ ), a $\mathrm{SiN}_{\mathrm{x}}$ or $\mathrm{SiGa}_{\mathrm{x}} \mathrm{N}_{\mathrm{y}}$ layer on the lateral surface was observed inhibiting the radial growth [27, 28]An ultra-thin contamination layer was also reported in core-shell multi QW wires [29-31] and it was shown by EDX analyses to be related to Si-enrichment [32-34]. This unintentional thin $\mathrm{SiGa}_{\mathrm{x}} \mathrm{N}_{\mathrm{y}}$ layer can be due to Si segragation at the surface [35].
Although the targeted doping level in the $\mathrm{n}-\mathrm{GaN}$ core part of the present LED was not exceeding $\sim 1 \times 10^{19} \mathrm{~cm}^{-3}$, we suspect that partial masking of the lateral surface may take place and could be responsible for the formation of a potential barrier at the core / underlayer interface.

Since the resistance of this interface layer may vary from wire to wire, its impact on the homogeneity of the light emission in the array can be significant. Namely, it can be the cause of the low NW EL yield observed on the array LED. In order to verify the impact of the potential barrier on the LED properties, two comparative analyses have been performed: $\mathrm{EBIC/CL}$ and EBIC/EL.

\section{EBIC/Cathodoluminescence correlated analyses}

Top view CL mapping has been performed on the NW array to analyse the homogeneity of the optical properties from wire to wire. In CL mapping, a spectrum is recorded for each position of the electron beam, hence building a hyperspectral map. By integrating the intensity of the luminescence linked to each pixel in different wavelength ranges, it is possible to obtain spectrally filtered maps [36-38]. The measurements were performed with an Attolight "Chronos" CL microscope with an acceleration voltage of $10 \mathrm{kV}$ and the spectra were recorded on an Andor Newton CCD camera with a Horiba dispersive spectrometer using a 150 grooves $/ \mathrm{mm}$ grating. Light is collected through an achromatic reflective objective with a numerical aperture of 0.7. The set-up is also equipped with an EBIC module allowing to simultaneously record the CL and EBIC maps.

To optimize the top view measurements, the ITO was removed from the top part of the NWs using a wet etch and the uncovered surface of the $\mathrm{p}-\mathrm{GaN}$ shell was exposed to a $\mathrm{CHF}_{3}$-based plasma to degrade the conductivity of the pdoped layer on top. This process allows for efficient excitation with the electron beam and at the same time preserves the full NW structure including the top part. To ascertain that the additional processing steps do not damaged the LED, its current-voltage curve and the EL spectra were measured as described in the supplementary information (cf. Figure 1S).

First, a CL map of a large area from the LED was recorded. Figure 3 displays the SEM image (a), the corresponding panchromatic CL map (b) and the CL spectrum recorded for the whole area (c). Contrary to the spotty EL, the CL map is quite homogeneous. Indeed, each NW produces a CL signal with relative wire-to-wire intensity fluctuations below $30 \%$, proving that there is no major difference in the non-radiative recombination from wire to wire. 

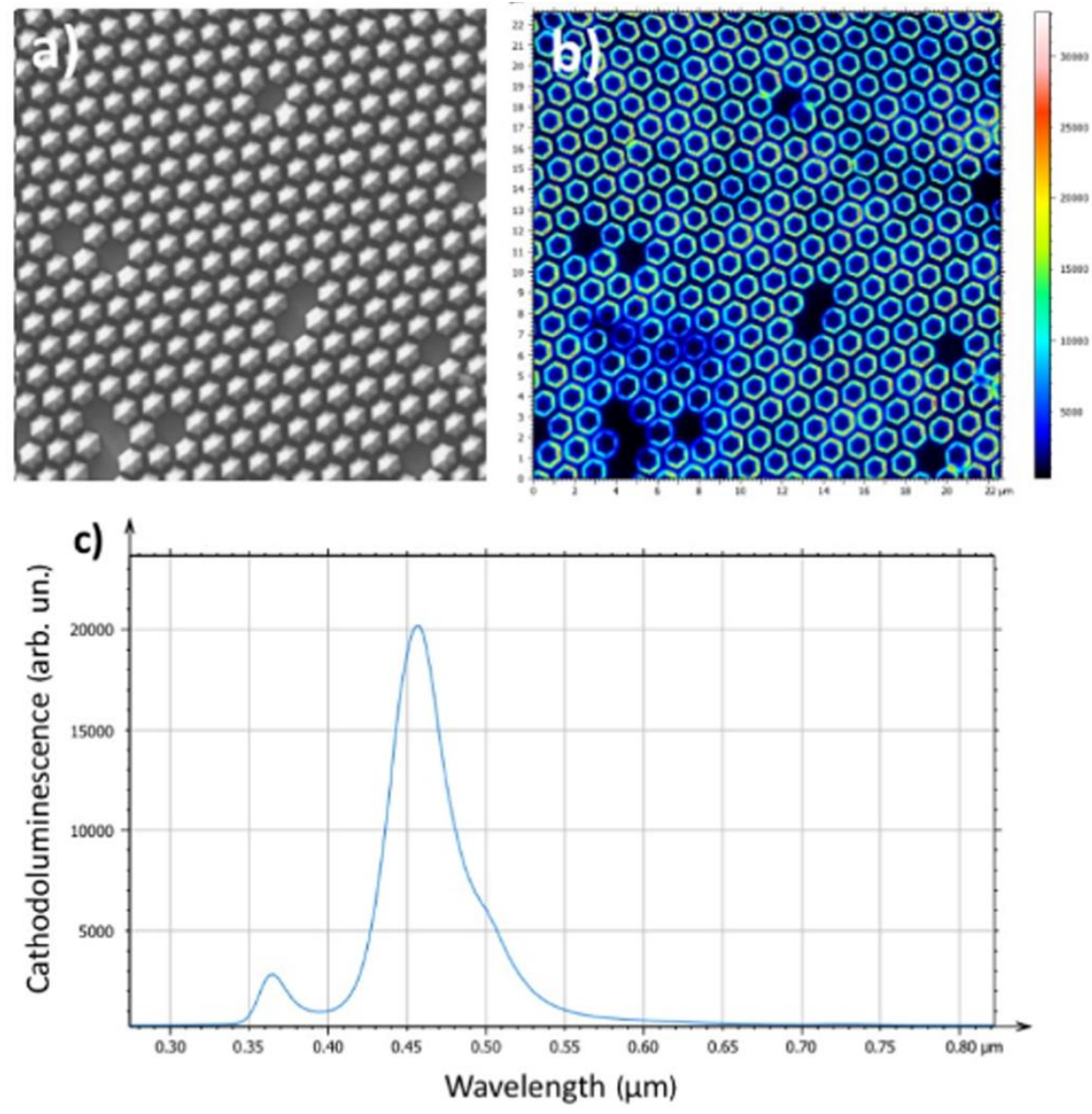
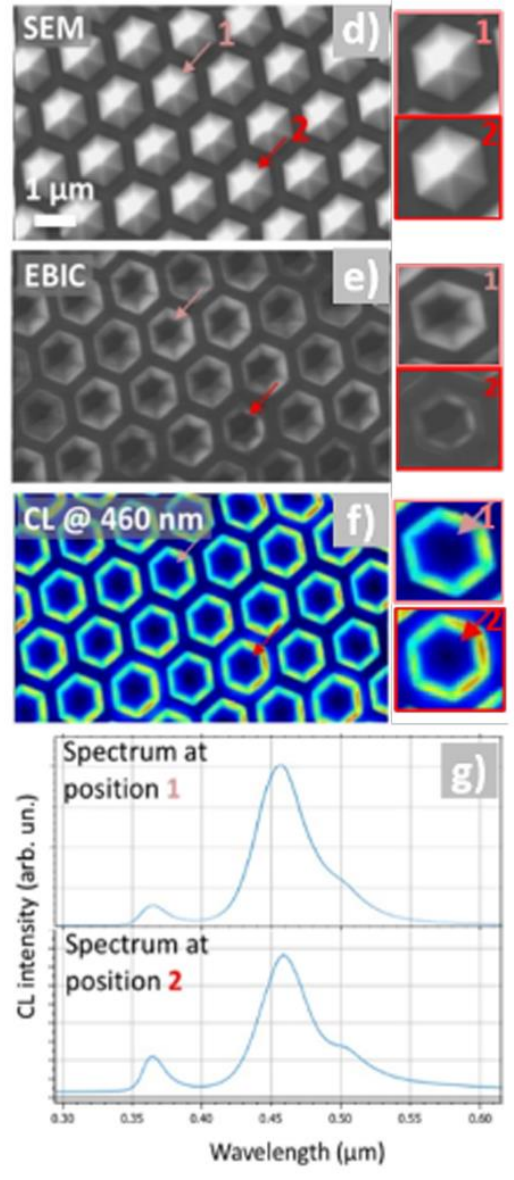

Figure 3: (a) Top-view SEM image and (b) the corresponding panchromatic CL map of the array. (c) CL spectrum of the whole area shown in panel (a). (d) SEM image, (e) EBIC map of a selected area from the array, (f) CL map integrated between 430 and $550 \mathrm{~nm}$. The area contains NWs with a normal and abnormal EBIC signal shown in the inset and labelled as "1" and " 2 ", respectively). (g) CL spectra of the NWs in positions 1 and 2, respectively.

Correlated EBIC / CL analyses were then performed at higher magnification as shown in Figure 3(d)-(g) (all measurements were performed under short circuit conditions). All NWs in this region have identical morphology (Figure 3(d)) and produce similar CL intensity (Fig. 3f). However, the EBIC map (Figure 3(e)) shows variations of the induced current. In particular, some NWs produce a weak signal located closer to the NW centre (which is referred to as "abnormal") which arises from the potential barrier at the core/underlayer interface, as it will be discussed in more detail in the next section. The CL properties of NWs with "normal" and "abnormal" EBIC signal were compared by acquiring the corresponding CL spectra. Two nanowires were selected highlighted by pink and red arrows, which exhibit respectively a normal EBIC signal (labelled as "1", in pink) and an abnormal signal (labelled as "2", in red). Despite the apparent difference in the charge collection, the NWs 1 and 2 produce luminescence of a similar intensity and spectral shape (Figure $3(\mathrm{~g}))$. The spectral analysis of the optical emission shows that for all the NWs irrespectively of their EBIC signature the luminescence is characterized by the GaN near band edge emission at $360 \mathrm{~nm}$, the emission from the m-plane QW at 465 $\mathrm{nm}$ and the green emission from the indium-rich region at 510 nm (Figure 3(c) and Figure 4(d)). The peak positions are in good agreement with the EL spectrum previously shown (Figure 1(e)).

The correlated CL/EBIC analyses prove that the optical properties of the array are highly homogeneous, while the charge collection properties are different. This indicates that the observed spotty EL is not related to the wire-to-wire fluctuations of the optical properties, but are induced by the difference in electrical properties.

\section{Top view EBIC mapping on "top-free" nanowires}

The EL and CL analyses both show the parasitic green emission. We have previously demonstrated that it can be suppressed by dry etching of the top part of the NWs [23]. The current-voltage $(\mathrm{I}(\mathrm{V}))$ curves and EL spectra of the LED after etching were assessed as discussed in the supplementary information (cf. Figure 1S). Although etching procedure preserves the EL, it may affect the electrical properties of the 
NWs and alter the internal potential barrier, object of our investigation. For this reason, top view EBIC measurements were performed on the NW arrays both before and after etching of the top semi-polar facets of the NWs. The SEM images and the corresponding EBIC maps of ITO-free full NWs are displayed in Figures 4(a)-(d), while SEM images and corresponding EBIC maps of etched "top-free" NWs are displayed in Figure 4(e)-(h).

In Figure 4(b) the majority of the NWs present a strong EBIC signal with a hexagonal shape (side of the hexagon $\sim 650$ $\mathrm{nm}$ ) corresponding to charge collection from the p-n junction. However, certain wires exhibit a weak EBIC signal which is located close to the center of the wire. Figure 4(d) shows a detail of the image where the two types of nanowires with the "normal" and "abnormal" EBIC signal are present. For the sake of clarity, all the NWs with an abnormal EBIC signal are marked by red arrows.

The abnormal signal can be better defined in the array where the tops of the nanowires were etched away (Figure $4(\mathrm{e})-(\mathrm{h}))$. In this case, there is no possible contribution from the semipolar facets. The regular EBIC signal in the "top-free" NWs presents a well-defined hexagonal shape (Figure 4(f)), whereas the abnormal signal manifests itself as a weak circular contrast (cf. Figure 4(h) for a high magnification image). The statistical analysis over several mesas showed that in both cases, there are $7-10 \%$ of nanowires with "abnormal" EBIC signal, indicating that the top NW etching does not affect the properties of the internal potential barrier causing the abnormal signal.

It should be noted that the "abnormal" EBIC signal in topview maps in the two cases has a larger diameter $(\sim 700-850$ $\mathrm{nm})$ than that of the nanowire core $(\sim 250-300 \mathrm{~nm})$. This is contrary to the case of the nanowires cleaved close to their base, where the "abnormal" signal follows the core boundary (cf. Figure 2(e)). We believe that this is due to the projection effect, namely to the broadening due to the interaction volume and the inhomogeneity of the signal along the wire axis. Indeed, for the situation shown in Figure 2(c), the widening of the top view signal can arise from the upper part of the nanowire.

\section{$\mathrm{EBIC/Electroluminescence} \mathrm{analyses}$}

For all the investigated configurations of the top LED contact the EL was strongly inhomogeneous, i.e. only $20-28$ $\%$ of NWs were producing electroluminescence. We note that the percentage of the NWs producing the EL was not degraded by the additional processing steps of ITO removal and top NW etching.

To evaluate the impact of the core/underlayer interface on the electro-optical behavior of the LED, top view EBIC maps were correlated with the optical microscopy images of the "top-free" LED under operation. The selected area was viewed in an optical microscope to visualize the EL from individual nanowires, then the same region was mapped by the EBIC microscopy. This order of experiments was chosen to avoid any possible modifications of the EL due to the electron beam exposure.

Figure 5 shows EL maps, SEM images and EBIC maps for the same area of the LED close to a metal contact frame. To establish the correlation between optical and scanning electron images, we have used the pattern defects (giving missing NWs), which are clearly seen in dark optical microscope images as well as in SEM images. Figure 5(a) shows a large area optical image whereas a higher magnification map of Figure 5(b) corresponds to the region explored in EBIC. As seen, there is a strong inhomogeneity of the EL from wire to wire: the EL intensity strongly fluctuates and some nanowires do not show any emission. The SEM image (Figure 5(c)) of the analysed region shows a regular nanowire morphology without any apparent pathology.

The EL maps in Figures 5(a) and (b) were recorded under low injection conditions (the applied bias of $4.0 \mathrm{~V}$ and the injected current density of $0.9 \mathrm{~A} / \mathrm{cm}^{2}$ ). To take into account a possible influence of the injection, similar analyses were performed for higher injection $\left(6.2 \mathrm{~V}, 4.0 \mathrm{~A} / \mathrm{cm}^{2}\right)$ as shown in the supplementary materials (Figure S2). The EL inhomogeneity is found to be almost independent from the injection conditions: the percentage of the active nanowires (i.e. the wires generating electroluminescence) at low injection is $\sim 20-25 \%$ and it increases only marginally at higher injection to 23-28\%.

The EBIC image (Figure 5(d)) presents some wire-to-wire fluctuations of the induced current, however it shows a current generation much more homogeneous than the EL signal. In particular, there is only $\sim 10 \%$ of nanowires that generate weak or no induced current.

The EBIC map allows to identify the nanowires with "abnormal" EBIC signal (marked with red arrows in Figure 5(d)), which can be then found on the EL map (indicated by red crosses in Figure 5(b)). A systematic analysis of the EL images evidences that each wire with "abnormal" EBIC behaviour shows no EL signal. However, the reverse is not true: a wire having a "normal" EBIC signal does not necessarily show EL.

In general, the number of nanowires producing EL $(\sim 20$ $28 \%$ ) is well below the number of nanowires having a "normal" EBIC signal ( $\geq 90 \%$ ).

The significant difference in the number of inactive nanowires in EBIC and EL experiments may stem from the difference in the operating conditions in the two measurements. This can be understood based on a simple electrical model of each individual NW as a diode with its individual series resistance. This series resistance represents the internal barrier at the core/underlayer interface. To illustrate the influence of this parameter on the EL and EBIC, simulated $\mathrm{I}(\mathrm{V})$ curves with varying access resistance are 
shown in Figure 5(e), where the EBIC and EL working conditions are highlighted. During EBIC measurements the induced current is collected in short circuit conditions, and therefore the signal dependence on the series resistance of the system is weak in a wide range of resistances. On the contrary, for EL the device is forward biased above the diode opening voltage and, in these conditions, the injected current strongly
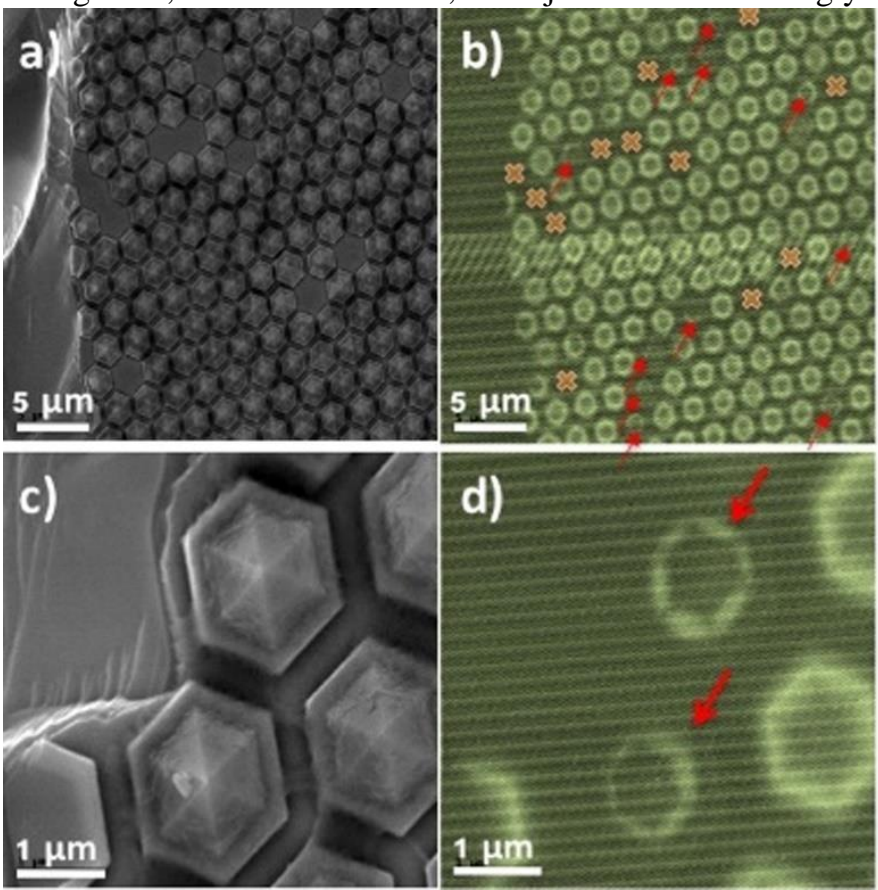

depends on the series resistance. These considerations applied to the EBIC and EL maps predict, that the EL variations due to internal potential barrier inhomogeneity are expected to be much stronger that the EBIC variations. To inhibit EBIC, a very high series resistance is required.
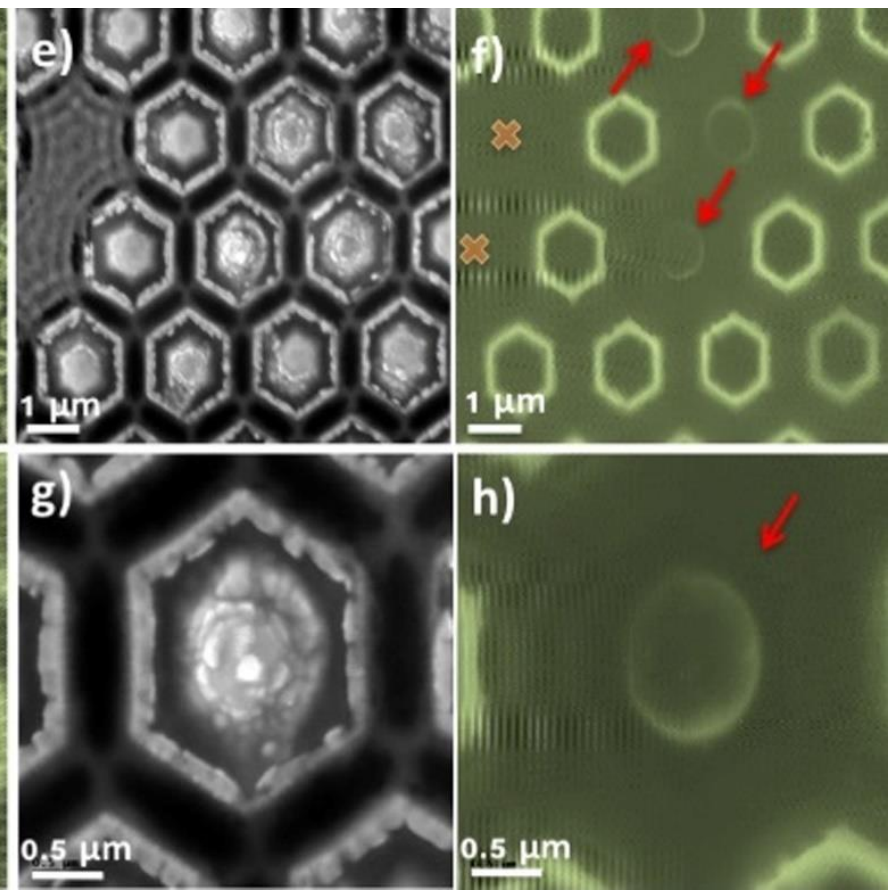

Figure 4. (a) SEM image and (b) the corresponding EBIC map of the LED with the ITO etch-back contact on top of nanowires. The crosses indicate the position of missing wires, the red arrows indicate nanowires with "abnormal" EBIC signal. (c) and (d) - the same sample as (a) and (b) under higher magnification showing a detail of the "abnormal" signal. (e) SEM image and (f) the corresponding EBIC image of the LED with ICP etching of the NW tops. (g) and (h) - the same sample as (e) and (f) under higher magnification showing a detail of the "abnormal" signal. All pictures and maps are acquired at $\mathrm{V}_{\mathrm{acc}}=10 \mathrm{kV}$.
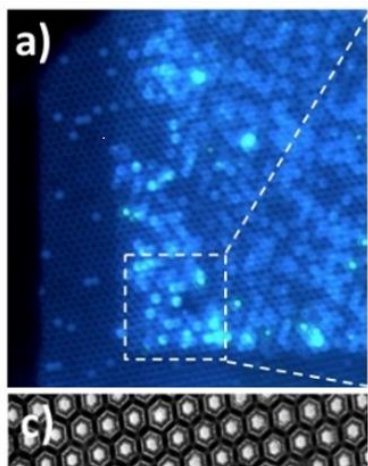
roogs nodo

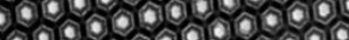

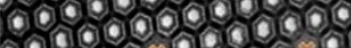
oog a organ rooo mogoros 10000000900 rooogoosorón

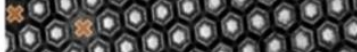

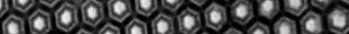
o0000000000 sing coopecos.

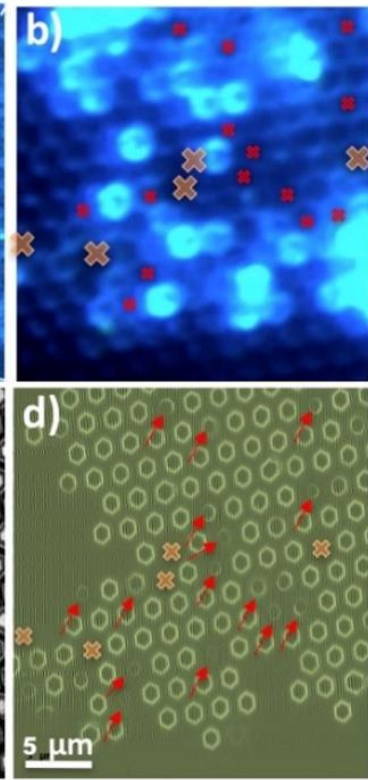

e)

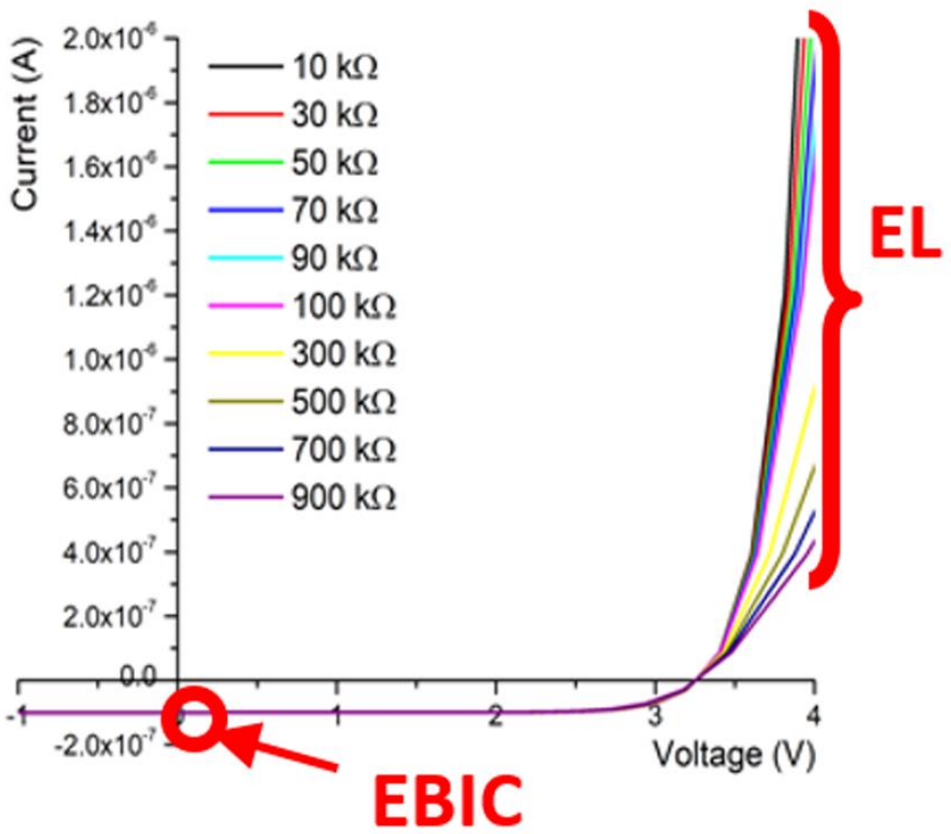


Figure 5. EL map of a region of the LED with etched NW tops for an applied voltage of $4 \mathrm{~V}$ and injected current density of $0.9 \mathrm{~A} / \mathrm{cm} 2$. (b) Higher-magnification image corresponding to the rectangle in panel (a). The pink crosses indicate the missing nanowires, the little red crosses indicate the position of nanowires producing an abnormal EBIC signal. (c) SEM image of the region shown with a rectangle in panel (a). The pink crosses show missing nanowires. (c) EBIC map corresponding to the panel (c). The red arrows point to the nanowires with abnormal EBIC signal. (e) Numerical simulation of the I(V) curve of a diode under excitation for different series resistances. The series resistance plays no significant role when measuring the EBIC current, while it strongly affects the carrier injection under forward bias and thus the EL intensity.

\section{Front $\mathrm{n}$-contact LED}

It is important to notice, that in all the EL experiments discussed so far, the electrons were injected from the $n-G a N$ NW core. In other terms, the electrical path to inject carriers in the QWs and to obtain EL involve the interface between the n-doped core grown in the axial direction and the n-doped $\mathrm{GaN}$ underlayer grown in the radial direction, where the potential barrier has been observed. The above-described analyses indicate that the LED operation is perturbed by the potential barrier at the core/underlayer interface, which is at the origin of the injection inhomogeneity. To prove this hypothesis and at the same time to overcome this problem, we developed an alternative LED architecture in which electrons are injected directly in the $\mathrm{n}-\mathrm{GaN}$ underlayer without crossing the core/underlayer interface ("front-n-contact" architecture). A new LED was processed from a different piece of the same growth wafer.

The fabrication steps of this "front n-contact" LED are illustrated in Figure 6. First, hole-injecting contact is defined to the NW sidewalls. A $200 \mathrm{~nm}$ ITO layer is deposited on the entire surface (Figure 6(a)) followed by a partial ITO etching from the upper part of the nanowires (Figure 6(b)). Then the $\mathrm{p}-\mathrm{GaN}$ layer is ICP-etched on the semipolar facets and on the upper part of the m-plane facets leaving the exposed n-GaN underlayer (Figure 6(c)).
The ITO layer forming the contact on the p-GaN shell is metallized on the sample edge to allow for the wire bonding (Figure 6(d)). The sample is then encapsulated into a hydrogen silsesquioxane (HSQ) layer to electrically insulate the contact to p-GaN shells (Figure 6(e)). Excess HSQ is etched with RIE. To contact the n-GaN underlayer, $300 \mathrm{~nm}$ ITO layer is deposited on top and patterned by lithography and lift-off processes. Finally, a Ti / Au metallization and lift-off are used to form the top metal contact for the bonding (Figure 6(f)). Panel $(\mathrm{g})$ illustrates the final structure of the front $\mathrm{n}$-contact LED, where the holes are injected through the ITO at lower part of the nanowires while the electrons are injected through the top ITO contact directly in the upper n-GaN underlayer (instead of being injected through the core). First, the electrical properties of the device were tested. We note that a high level of leakage is observed for reverse bias as shown in Figure 7(a). It is possible that the leakage problem arises from a parasitic conduction between the first ITO layer and the n$\mathrm{GaN}$ underlayer. Indeed, after the ICP etching of the upper part of the nanowire, the ITO surpasses the edge of the bottom part of p-GaN shell and it is not mechanically supported by the nanowire. At the time of HSQ encapsulation, the ITO layer can break and touch the n-GaN layer creating local short circuits. 
(a) bottom ITO contact
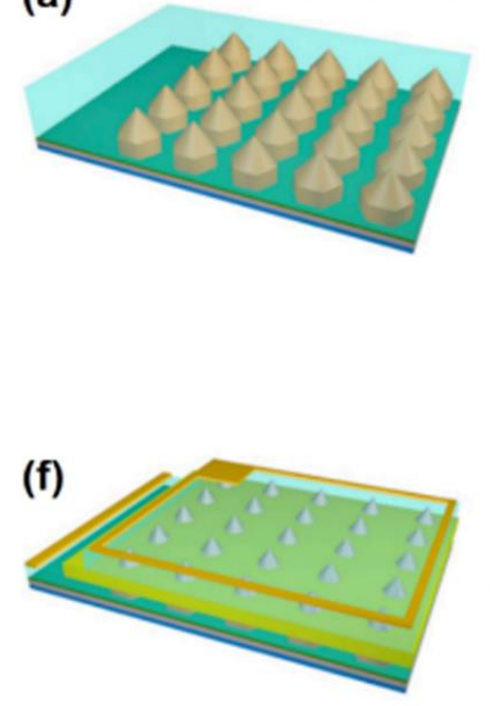
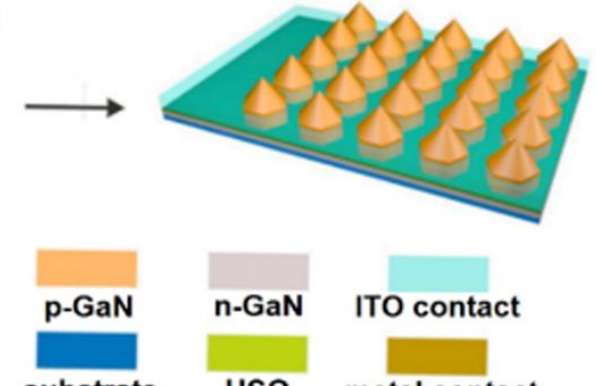

(b)

ITO etch back

(e)

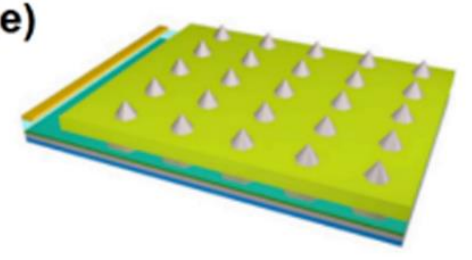

HSQ encapsulation

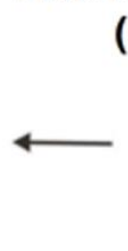

(c) top part p-GaN etching
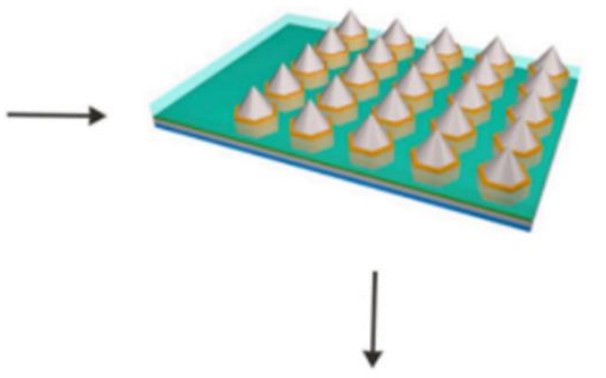

(d)
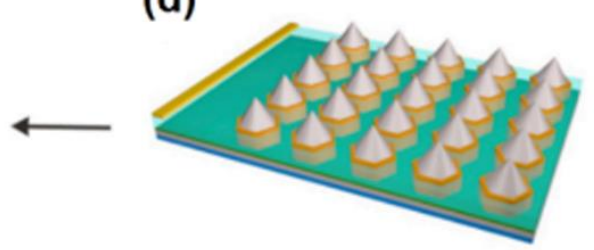

metal contact for $\mathrm{p}-\mathrm{GaN}$

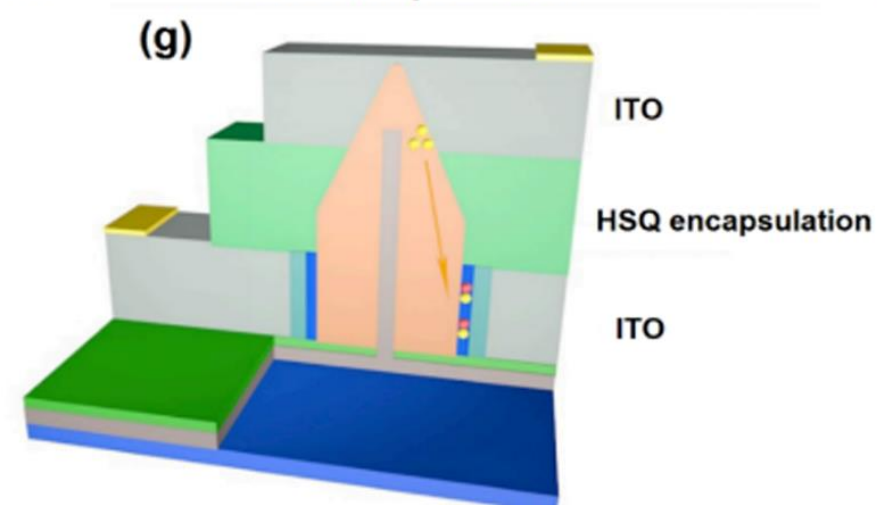

Figure 6. Font n-contact LED manufacturing steps: (a) deposition of the ITO for the hole injection. (b) partial etching of the ITO. (c) ICP etching of the p- GaN shell. (d) definition of a metal contact to the ITO layer. (e) encapsulation with the HSQ and its partial etching. (f) lithography and deposition of the ITO and the metal contact for the electron injection. (d) Schematic of the final device.

This leakage is also present under forward bias, which results in a much higher operation current density in comparison to the previously described EL measurements of the conventional LED.

Despite the high leakage current, the front n-contact LED produces EL starting from an applied voltage of 3.3 V. This number is the same as for LEDs with the electron injection through the $\mathrm{n}-\mathrm{GaN}$ core. The EL spectra for an increasing current injection are shown in Figure 7(b). As expected, the emission of the front $n$-contact LED presents a single peak at $2.6 \mathrm{eV}(476 \mathrm{~nm})$, which remains stable with increasing current.

Finally, the homogeneity of the EL maps is compared between the standard LEDs where the electrons injected through the core/underlayer interface and the front n-contact LED where the electrons are directly injected in the $n-G a N$ underlayer. As for the previous devices, a statistical analysis is performed on the front n-contact LED operated under a bias of $4 \mathrm{~V}$. The examples of the EL maps for the LEDs discussed in this paper are shown in Figure 8(a)-(c), respectively corresponding to the entire NWs with a full ITO contact (a), to "top-free" NWs (b) and to "front-n-contact" NWs (c). Comparing these maps, a significant increase in the NWs yield can be observe, going from $19 \%-22 \%$ for the first two cases up to $65 \%$ in the front-n-contact device. This result demonstrates that the electron injection directly in the n-GaN underlayer strongly improves the homogeneity of the EL, i.e. that the presence of a potential barrier at the core/underlayer interface increases the access resistance when injecting electrons into the active region[22].

Contextually, it can also be noted that front-n-contact architecture is effectively reducing the homogeneity issue at a larger scale, as shown on the EL map taken with a lower magnification in Figure 8(d). It is possible that the EL homogeneity may be further improved by optimizing the 
fabrication processes. Indeed, the map of Figure 8(d) shows that most of the inactive nanowires are gathered together in localized areas, which may indicate process problems in these regions.
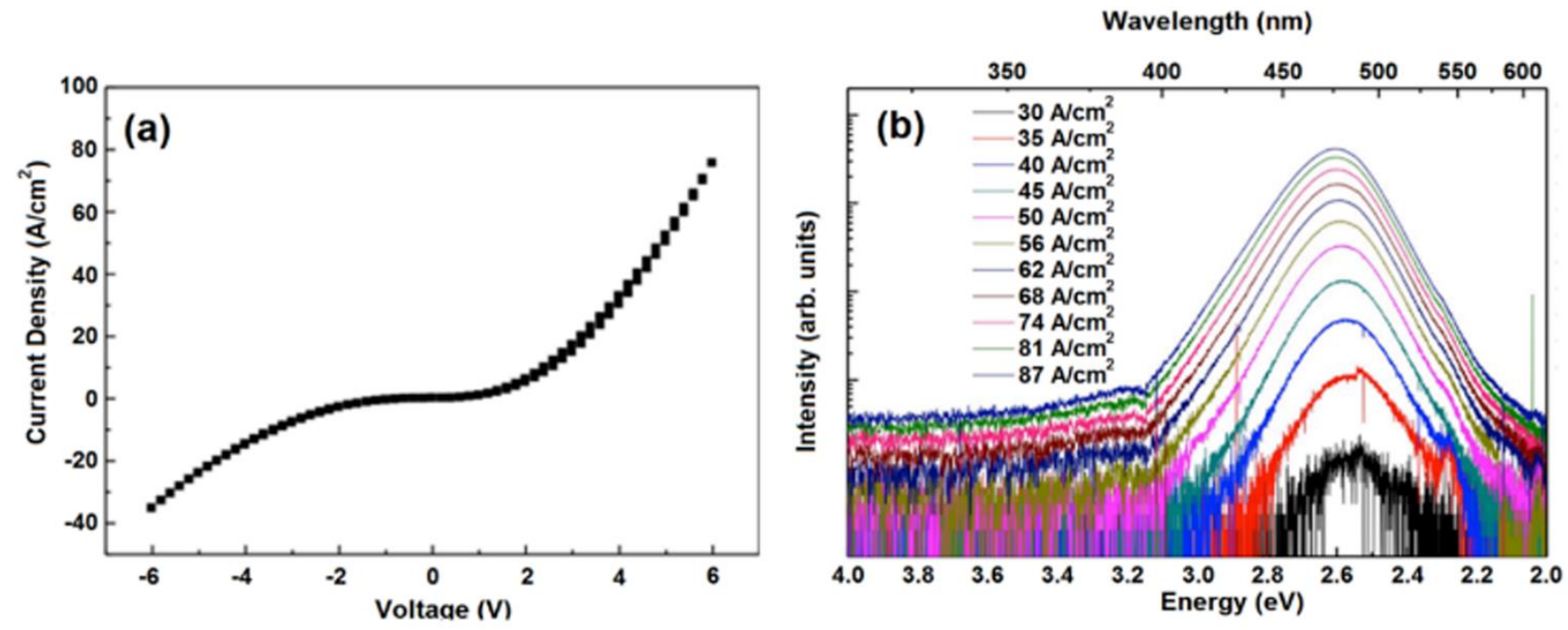

Figure 7. (a) J-V curve of the front n- contact LED. (b) EL spectra of the front n-contact LED for increasing injection.

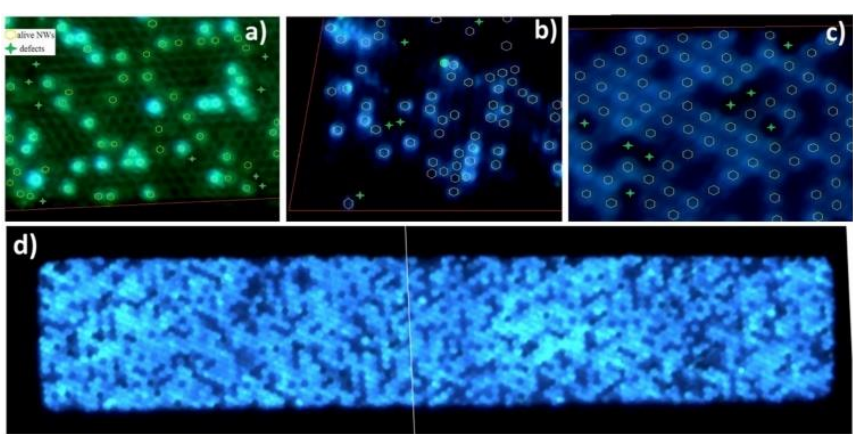

Figure 8. EL maps for LED processes with (a) injection of electrons through the core and a conformal top ITO contact. (b) injection of electrons through the core and the etched upper part of nanowires. (c) front n-contact LED and electrons injected directly in the $n-G a N$ underlayer. (d) Intermediate magnification of EL map showing emission fluctuations over a large area.

\section{Conclusions}

In the present work, we analysed the injection inhomogeneity in core-shell InGaN/GaN NW LEDs. We concluded that in the investigated case it is mainly induced by a potential barrier at the interface between the nanowire n$\mathrm{GaN}$ core and the radially grown $\mathrm{n}-\mathrm{GaN}$ layer. CL analyses showed that the optical properties of the nanowires are homogeneous. The comparison of EL and EBIC maps on the same region offered a proof that the potential barrier hindered the homogeneous charge carrier injection in different wires and reduced the efficiency of the nanowire LED array. In order to avoid injection through a potential barrier at the core/underlayer interface, we changed the contact architecture and fabricated the "front contacted" LED, for which the electrons are injected directly into the $\mathrm{n}-\mathrm{GaN}$ underlayer. The "front contacted" LED presented an increased yield of the NWs producing the EL (the yeild was increased from $\sim 20 \%$ for standard contact to $65 \%$ for front contact). This proves that the front electron injection allows to reduce the impact of the potential barrier at the core/underlayer interface.

\section{Acknowledgements}

This work has been partially financially supported by EU ERC project 'NanoHarvest' (grant no. 639052), by the Region Ile-de-France in the framework of C'Nano IdF (the nanoscience competence center of the Paris region), by the European Union (FEDER 2007-2013), and by the Labex GANEX (grant no. ANR-11-LABX-0014) as part of the "Investissements d'Avenir" program managed by the French National Research Agency (ANR). The authors acknowledge O. Kryliuk for providing nanowire samples.

\section{ORCID iDs}

Andrey Babichev https://orcid.org/0000-0002-3463-4744

Maria Tchernycheva https://orcid.org/0000-0003-4144-0793

\section{References}

[1] Guan N, Dai X, Julien F H, Eymery J, Durant C and Tchernycheva M 2019 Nitride Nanowires for Light Emitting Diodes. Light-Emitting Diodes. Materials, Processes, Devices and Applications 425-84 https://doi.org/10.1007/978-3-31999211-2_12

[2] Robin Y, Bae S Y, Shubina T V, Pristovsek M, Evropeitsev E A, Kirilenko D A, Davydov V Yu, Smirnov A N, Toropov A A Jmerik V N, Kushimoto M, Nitta S, Ivanov S V and Amano H 
2018 Insight into the performance of multi-color InGaN/GaN nanorod light emitting diodes Sci. Rep. 87311

[3] Kapoor A, Guan N, Vallo M, Messanvi A, Mancini L, Gautier E, Bougerol C, Gayral B, Julien F H, Vurpillot F, Rigutti L, Tchernycheva M, Eymery J and Durand C 2018 Green Electroluminescence from Radial m-Plane InGaN Quantum Wells Grown on GaN Wire Sidewalls by Metal-Organic Vapor Phase Epitaxy ACS Photonics 5 4330-7

[4] Schmidt G, Müller M, Veit P, Metzner S, Bertram F, Hartmann J, Zhou H, Wehmann H-H, Waag A and Christen J 2018 Direct imaging of Indium-rich triangular nanoprisms self-organized formed at the edges of InGaN/GaN core-shell nanorods Sci. Rep. 816026

[5] Yi W, Uzuhashi J, Chen J, Kimura T, Kamiyama S, Takeuchi T, Ohkubo T, Sekiguchi T and Hono K 2019 Cathodoluminescence and scanning transmission electron microscopy study of InGaN/GaN quantum wells in core-shell GaN nanowires Appl. Phys. Express 12085003

[6] Motohisa J, Kameda H, Sasaki M and Tomioka K 2019 Characterization of nanowire light-emitting diodes grown by selective-area metal-organic vapor-phase epitaxy Nanotechnology 30134002

[7] Ra Y-H and Lee C-R 2019 Understanding the p-Type GaN Nanocrystals on InGaN Nanowire Heterostructures ACS Photonics 6 2397-404

[8] Bi Z, Lenrick F, Colvin J, Gustafsson A, Hultin O, Nowzari A, Lu T, Wallenberg R, Timm R, Mikkelsen A, Ohlsson B J, Storm K, Monemar B and Samuelson L 2019 InGaN Platelets: Synthesis and Applications toward Green and Red LightEmitting Diodes Nano Lett. 19 2832-9

[9] Nami M, Rashidi A, Monavarian M, Mishkat-Ul-Masabih S, Rishinaramangalam A K, Brueck S R J and Feezell D 2019 Electrically Injected GHz-Class GaN/InGaN Core- NanowireBased $\mu$ LEDs: Carrier Dynamics and Nanoscale Homogeneity ACS Photonics 6 1618-25

[10] Hartensveld M, Zhang J 2019 Monolithic Integration of GaN Nanowire LightEmitting Diode With Field Effect Transistor IEEE Electron Device Letters 40427

[11] Li S and Waag A 2012 GaN based nanorods for solid state lighting J. Appl. Phys. 111071101

[12] Kang M S, Lee C-H, Park J B, Yoo H and Yi G-C. 2012 Gallium nitride nanostructures for light-emitting diode applications Nano Energy 1 391-400

[13] Chung K, Beak H, Tchoe Y, Oh H, Yoo H, Kim M and Yi G-C 2014 Growth and characterizations of GaN micro-rods on graphene films for flexible light emitting diodes APL Mater. 2 092512

[14] Dai X, Messanvi A, Zhang H, Durand C, Eymery J, Bougerol C, Julien F H and Tchernycheva M 2015 Flexible LightEmitting Diodes Based on Vertical Nitride Nanowires Nano Lett. 15 6958-64

[15] Koester R, Hwang J-S, Salomon D, Chen X, Bougerol C, Barnes J-P, Dang D L S, Rigutti L, de Luna Bugallo A, Jacopin G, Tchernycheva M, Durand C and Eymery J 2011 M-Plane Core-Shell InGaN/GaN Multiple-Quantum-Wells on GaN Wires for Electroluminescent Devices Nano Lett. 11 4839-45

[16] Jacopin G, De Luna Bugallo A, Lavenus P, Rigutti L, Julien F H, Zagonel L F, Kociak M, Durand C, Salomon D, Chen X J,
Eymery J and Tchernycheva M 2011 Single-Wire LightEmitting Diodes Based on GaN Wires Containing Both Polar and Nonpolar InGaN/GaN Quantum Wells Appl. Phys. Express 5014101

[17] Li S, Wang X, Fündling S, Erenburg M, Ledig J, Wei J, Wehmann H H, Waag A, Bergbauer W, Mandl M, Strassburg M, Trampert A, Jahn U, Riechert H, Jönen H and Hangleiter A 2012 Nitrogen-polar core-shell GaN light-emitting diodes grown by selective area metalorganic vapor phase epitaxy. Appl. Phys. Lett. 101032103

[18] Chen H-S, Yao Y-F, Liao C-H, Tu C-G, Su C-Y, Chang W-M, Kiang Y-W and Yang C C 2013 Light-emitting device with regularly patterned growth of an $\mathrm{InGaN} / \mathrm{GaN}$ quantum-well nanorod light-emitting diode array Opt. Lett. 383370

[19] Tchernycheva M, Lavenus P, Zhang H, Babichev A V, Jacopin G, Shahmohammadi M, Julien F H, Ciechonski R, Vescovi G and Kryliouk O 2014 InGaN/GaN Core-Shell Single Nanowire Light Emitting Diodes with Graphene-Based P-Contact Nano Lett. 14 2456-65

[20] Chang J-R, Chang S-P, Li Y-J, Cheng Y-J, Sou K-P, Huang JK, Kuo H-C and Chang C-Y 2012 Fabrication and luminescent properties of core-shell InGaN/GaN multiple quantum wells on GaN nanopillars Appl. Phys. Lett. 100261103

[21] Hong Y J, Lee C-H, Yoon A, Kim M, Seong H-K, Chung H J, Sone C, Park Y J and Yi G-C 2011 Visible-Color-Tunable Light-Emitting Diodes Adv. Mater. 23 3284-8

[22] Tchernycheva M, Neplokh V, Zhang H, Lavenus P, Rigutti L, Bayle F, Julien F H, Babichev A, Jacopin G, Largeau L, Ciechonski R, Vescovi G and Kryliouk O 2015 Core-shell InGaN/GaN nanowire light emitting diodes analyzed by electron beam induced current microscopy and cathodoluminescence mapping Nanoscale 7 11692-701

[23] Zhang H, Jacopin G, Neplokh V, Largeau L, Julien F H, Kryliouk O and Tchernycheva M 2015 Color control of nanowire InGaN/GaN light emitting diodes by post-growth treatment Nanotechnology 26465203

[24] Lavenus P, Messanvi A, Rigutti L, De Luna Bugallo A, Zhang H, Bayle F, Julien F H, Eymery J, Durand C and Tchernycheva M 2014 Experimental and theoretical analysis of transport properties of core-shell wire light emitting diodes probed by electron beam induced current microscopy. Nanotechnology $\mathbf{2 5}$ 255201

[25] Tanaka S, Takeuchi M and Aoyagi Y 2000 Anti-Surfactant in III-Nitride Epitaxy - Quantum Dot Formation and Dislocation Termination - Jpn. J. Appl. Phys. 39 L831-4

[26] Rosa A L, Neugebauer J, Northrup J E, Lee C-D and Feenstra R M 2008 Adsorption and incorporation of silicon at GaN(0001) surfaces Appl. Phys. Lett. 80 2008-10

[27] Markurt T, Lymperakis L, Neugebauer J, Drechsel P, Stauss P, Schulz T, Remmele T, Grillo V, Rotunno E and Albrecht M 2013 Blocking growth by an electrically active subsurface layer: the effect of $\mathrm{Si}$ as an antisurfactant in the growth of GaN. Phys. Rev. Lett. 110036103

[28] Eymery J, Salomon D, Chen X J and Durand C 2012 Process for Catalyst-Free Selective Growth on a Semiconductor Structure Patent WO2012136665

[29] Mandl M, Wang X, Schimpke T, Kölper C, Binder M, Ledig J, Waag A, Kong X, Trampert A, Bertram F, Christen J, Barbagini F, Calleja E and Strassburg M 2013 Group III nitride core-shell 
nano- and microrods for optoelectronic applications Phys. Status Solidi RRL 7 800-14

[30] Yi W, Uzuhashi J, Chen J, Kimura T, Kamiyama S, Takeuchi T, Ohkubo T, Sekiguchi T and Hono K 2019

Cathodoluminescence and scanning transmission electron microscopy study of InGaN/GaN quantum wells in core-shell GaN nanowires Appl. Phys. Express 12085003

[31] Durand C, Bougerol C, Carlin J-F, Rossbach G, Godel F, Eymery J, Jouneau P-H, Mukhtarova A, Butté R and Grandjean N 2014 M-Plane GaN/InAlN Multiple Quantum Wells in CoreShell Wire Structure for UV Emission ACS Photonics 1 38-6

[32] Tessarek C, Heilmann M, Butzen E, Haab A, Hardtdegen H, Dieker C, Spiecker and Christiansen S 2014 The Role of Si during the Growth of GaN Micro- and Nanorods Cryst. Growth Des. 14 1486-92.

[33] Ren C X, Tang F, Oliver R A and Zhu T 2018 Nanoscopic insights into the effect of silicon on core-shell $\mathrm{InGaN} / \mathrm{GaN}$ nanorods: Luminescence, composition, and structure J. Appl. Phys. 123045103

[34] Tessarek C, Rechberger S, Dieker C, Heilmann M, Spiecker E and Christiansen S 2017 Understanding GaN/InGaN core-shell growth towards high quality factor whispering gallery modes from non-polar InGaN quantum wells on $\mathrm{GaN}$ rods Nanotechnology 28485601

[35] Kapoor A, Finot S, Grenier V, Robin E, Bougerol C, Bleuse J, Jacopin G, Eymery J and Durand C 2020 Role of Underlayer for Efficient Core-Shell InGaN QWs Grown on m-plane GaN Wire Sidewalls ACS Appl. Mater. Interfaces 12 19092-101

[36] Piazza V, Babichev A V, Mancini L, Morassi M, Quach P, Bayle F, Largeau L, Julien F H, Rale P, Collin S and Harmand J C 2019 Investigation of GaN nanowires containing AlN/GaN multiple quantum discs by EBIC and CL techniques Nanotechnology 30214006

[37] Chen H L, Himwas C, Scaccabarozzi A, Rale P, Oehler F, Lemaître A, Lombez L, Guillemoles J F, Tchernycheva M, Harmand J C and Cattoni A 2017 Determination of n-type doping level in single GaAs nanowires by cathodoluminescence Nano Lett. 17 6667-75

[38] Zagonel LF, Mazzucco S, Tencé M, March K, Bernard R, Laslier B, Jacopin G, Tchernycheva M, Rigutti L, Julien F H and Songmuang R 2011 Nanometer scale spectral imaging of quantum emitters in nanowires and its correlation to their atomically resolved structure Nano Lett. 11 568-73 